\title{
Effects of Dietary Supplementation of Soy Isoflavones on the Performance and Egg Quality in Native Chinese Breeder Hens
}

\section{-Author(s)}

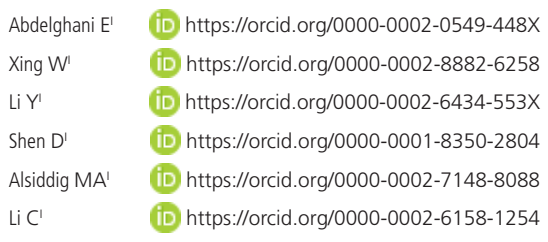

College of Animal Science and Technology, Nanjing Agricultural University, Nanjing 210095, P.R. China

\section{-Mail Address}

Corresponding author e-mail address Chunmei Li

College of Animal Science and Technology, Nanjing Agricultural University, Nanjing

210095, P.R. China.

Phone: +86-025-84395971

Email: chunmeili@njau.edu.cn

\section{-Keywords}

Xueshan hens, egg quality, hatching traits, organs weight, cholesterol.

\section{ABSTRACT}

To investigate the effects of soy isoflavones (ISF) on the laying performance of Xueshan breeder hens at different phases of the egg production cycle, 32 weeks (wks) old local Chinese breeder hens were assigned to two groups with three replicates (750 hens/replicate) for control and treatment groups. Then every group was divided into three sub-groups following 4, 12, and 20 wks administration of control or ISF diet. General Linear Model procedure was used $(2 \times 3)$, and treatments were considered of two factors (diets and time) with different levels (control and ISF diets; 4, 12, and 20 wks of diet supply time). Basal diet with $3 \mathrm{mg} / \mathrm{kg}$ ISF was used. Performance, egg quality, organs relative weight and cholesterol level were measured at three time-points of 36, 44 , and 52 -wks of age old. The results showed that both diets and time significantly affected the production performance and physiological parameters. In details, ISF inclusion significantly $(p=0.02)$ decreased average egg weight and average feed intake compared to control at 52wks of age old, however, feed conversation ratio was not affected by ISF diet. Moreover, ISF diet caused to an increase hatching rate $(p=0.01)$ at 36-wks old hens, an abnormal egg rate decrease $(p=0.02)$ at 44 -wks old, an increase in yolk color $(p=0.035)$ and Haugh unit $(p=0.039)$, and a decrease in low-density lipoprotein cholesterol level $(p=0.03)$ at 52 wks of age old. ISF diet also increased spleen relative weight $(p=0.041)$ and total antioxidant capacity compared to the control group. The results revealed that ISF inclusion exerted some benefits, thus it could be a potential feed additive for the breeder hens.

\section{INTRODUCTION}

Feed additives of plant origin gain more interest among producers and nutritionists due to their safety, availability, their possible role in improving growth performance due to their antioxidant properties, and the lack of side effects for human and animals as well. Soy isoflavones (ISF) are natural synthesized phenolic compounds plants, which exist in two forms: conjugated or unconjugated (Kudou et al., 1991). They are commonly known as phytoestrogens because of their structural similarity to $17 \beta$-estradiol, and their capability to bind to estrogen receptor (ERs) with much more affinity to ER $\beta$ (Pilsakova et al., 2010). Soybean is considered to be the richest source of isoflavones.

During the last decade, scientists paid more attention to ISF and their compound (daidzein, genistein, and glycitein) due to their noticeable positive effects in human health (Ajdžanović et al., 2014). In addition, several studies showed that ISF had an antioxidant property (Jiang et al., 2007), could promote immune function (Zhang et al., 1997), prevented the breast cancer, lowered the risk of osteoporosis, decreased the plasma cholesterol level, and enhanced the antioxidative potential in humans and animals (Messina, 2010). 
ISF has recently become popular as a dietary supplementation, especially for late laying production stage as a hormonal substitute to enhance the egg production rate. It is suggested that endogenous estrogen state, individual variation, duration and dose of phytoestrogen affects its act as an estrogen agonist or antagonist (Cassidy, 2003). Several studies reported that ISF improved growth and reproductive performance of the animals and their products (Yousef et al., 2004; Jiang et al., 2007; Shin et al., 2008). Furthermore, Shi et al. (2013) reported that feeding ISF to laying hens at 59 weeks of age showed an increase in egg production. Moreover, dietary ISF daidzein at 10, 20 , and $30 \mathrm{mg} / \mathrm{kg}$ improved fertility and egg weight (Zhao et al., 2013). Also, a diet supplemented with ISF daidzein for Shaoxing duck breeder significantly enhanced the productive performance during late laying stage (Zhao et al., 2005), and the same improved laying performance, and eggshell quality were observed in post-peak laying stage of hens ( $\mathrm{Ni}$ et al., 2007). Moreover, feeding quail in the late laying stage significantly improved egg quality and mineralization of the bone (Sahin et al., 2007).

Calcium (Ca) plays a vital role in bone and eggshell formation. ISF had been shown to decrease $\mathrm{Ca}$ concentration in osteoclasts (Kajiya et al., 2000), and consequently increase the available $\mathrm{Ca}$ for eggshell formation. Studies conducted by Zhao et al. (2005) and Sahin et al. (2007) confirmed that using ISF daidzein improved eggshell in chicken and quail, respectively.

It is extremely important to obtain a maximum number of eggs with optimal internal and external characteristics in breeder hens, especially for normal embryo development (Kontecka et al., 2012). On the other hand, broiler breeder diets modulate subsequent egg production rate (Peebles et al., 2000), embryo development and egg hatchability (Peebles et al., 2000).

Xueshan 'snow mountain' is a local hybrid meat type, it's a favorite among consumers for its delicate, tender and delicious meat properties. Therefore, as far as we know, studies on the effect of ISF on performance and egg quality in domestic broiler breeder hens are limited. In general, the effects of ISF on poultry are contradictory. This study aimed to determine the effect of long term ISF supplementation on egg production, egg quality, fertility, hatchability, and cholesterol level at 36 wk, 44 wk, and 52 wk of age in local Chinese breeder hens.

\section{MATERIALS AND METHODS}

\section{Birds and experimental treatments}

Four thousand five hundred (4500), 32-week-old Chinese local Xueshan breeder hens were randomly divided into two groups: control and treatment groups. Each group consisted of three replicates and each replicate had 750 birds. Each group was divided into three sub-groups following 4, 12, and 20 wks administration of control or ISF diet. The experiment design followed General Linear Model procedure (2 $\times 3)$, and treatments were considered of two factors (diets and time) with different levels (control and ISF diets; 4, 12, and 20 wks of diet supply time). The birds in the control group were fed with a basal diet, while birds in the experimental group were fed with basal diet+ $3 \mathrm{mg} / \mathrm{kg}$ soy ISF from 28 to $52 \mathrm{wk}$ of age. The trial lasted for 20 weeks. Every five days breeder hens were inseminated with $25 \mu \mathrm{l}$ of semen from cocks that received basal diets. Laying performance, egg quality, hatchability, and fertility were measured at 36 wk, $44 w k$, and $52 w k$, respectively. After the birds were slaughtered, organs (ovary, oviduct, heart, spleen, and kidney) were dissected and immediately weighed.

This trial was conducted in Jiangsu Lihua Animal Husbandry Stock Co. Ltd (Jiangsu, China). Soy isoflavone was obtained from Lihua Industrial Corporation (Changzhou, China). The birds were housed in wire cages, under $16 \mathrm{~h}$ light: $8 \mathrm{~h}$ dark cycle, and controlled temperature of $21^{\circ} \mathrm{C}$. All birds were fed with $130 \mathrm{~g} / \mathrm{bird} /$ day, and free access to water. The composition and nutrient contents of the experimental diets are presented in Table 1. All animal handling protocols were approved by the Animal Care and Use Committee of Nanjing Agricultural University.

\section{Performance and egg quality}

Eggs were collected twice a day, and egg production rate was calculated from the total number of eggs divided by the total number of days. Feed intake was recorded on a replicate basis at weekly intervals. Egg mass was calculated as egg production rate $x$ average egg weight. Feed conversion ratio was calculated as a gram of feed consumed divided by a gram of egg produced.

For egg quality, six eggs were chosen randomly at the age of $36 w k, 44 w k$, and $52 w k$ respectively and analyzed for internal and external characteristics. The length and width were measured using Digital Caliper $^{\circledR}$ (MNT-150. Shanghai, China). The shape index was calculated (width/length $\times 100$ ). Egg Multi-tester 
Table 1 - Chemical composition and nutrient of the basal diet.

\begin{tabular}{lc}
\hline Ingredients & Composition (\%) \\
\hline Corn & 64.1 \\
Soybean meal & 19.0 \\
Fish meal & 2.0 \\
Limestone & 8.6 \\
Bran & 3.7 \\
Sodium chloride & 0.3 \\
Dicalcium phosphate & 1.3 \\
Premix & 1.0 \\
Total & 100.0 \\
\hline Calculated composition ${ }^{2}$ & \\
ME (MJ/kg) & 11.17 \\
CP3 (\%) & 16.16 \\
Cys +Met & 0.59 \\
Lys (\%) & 0.92 \\
Calcium (\%) & 3.56 \\
AP (\%) & 0.38 \\
\hline
\end{tabular}

1 Provided per kilogram of diet: vitamin A, 6,000 IU; vitamin D3, 3,630 IU; vitamin E, $22 \mathrm{IU}$; vitamin K $2.2 \mathrm{mg}$; vitamin B1 $2.2 \mathrm{mg}$; vitamin B2 $8.25 \mathrm{mg}$; vitamin B6 $6.6 \mathrm{mg}$ biotin $0.55 \mathrm{mg}$; folic acid $1.1 \mathrm{mg}$; vitamin B12 $0.011 \mathrm{mg}$; vitamin B3 $20 \mathrm{mg}$; copper, 15 $\mathrm{mg}$; iron, $70 \mathrm{mg}$; zinc, $75 \mathrm{mg}$; manganese, $50 \mathrm{mg}$; iodine, $0.55 \mathrm{mg}$; selenium, $0.36 \mathrm{mg}$. ${ }^{2} \mathrm{ME}$, metabolic energy; $\mathrm{CP}$, crude protein; Cys, cysteine; Met, methionine; Lys, Iysine; $\mathrm{AP}$, available phosphorus.

(EMT-5200, Crescent Science and Technology Co. Ltd. China) was used to automatically measure egg weight, albumen height, Haugh unit (HU), and yolk color. The device uses 1 throughout of 15 scales color index to distinguish the yolk color density, ultrasound sensors to measure albumen height. The yolk was separated from the albumen and then weighed. The eggshell was weighed with the shell membrane. The ratio of yolk to albumen was calculated.

\section{Fertility and hatchability}

Eggs for hatching were collected at 36 wk, 44wk, and $52 \mathrm{wk}$ and stored at $18{ }^{\circ} \mathrm{C}$ before incubated. The abnormal egg rate was calculated as number of abnormal eggs/number of laying eggs $\%$, soft egg rate was determined as number of soft eggs/number of laying eggs $\%$. The thermal condition for the incubator was $37^{\circ} \mathrm{C}$ and $60 \%$ relative humidity. Incubated eggs were candled on the $7^{\text {th }}$ day of incubation to determine the number of infertile eggs. The Fertility \% was calculated as number of fertile egg $\times 100$ /number of eggs set. On day 21 the hatchability of fertile eggs was calculated.

\section{Sample collection}

Hens were weighed individually at 36, 44, and 52 wks of age to evaluate body weight. Eight birds from each time-period were slaughtered to collect blood samples, which immediately were centrifuged $(3,000$ $\times \mathrm{g}$ at $\left.4^{\circ} \mathrm{C}\right)$, and the isolated serum was stored at -20 ${ }^{\circ} \mathrm{C}$ until further analysis. Organs (ovary, oviduct, heart, spleen, liver, and kidney) were dissected and weighed.

\section{Cholesterol assay}

Serum samples were examined for total cholesterol (TCHO), low-density lipoprotein cholesterol (LDL-C), high-density lipoprotein cholesterol (HDL-C), triglycerides (TG), and total antioxidant capacity (T-AOC) using a commercial kit according to manufacturer's instructions (Jian Cheng Bioengineering Institute, Nanjing, China).

\section{Statistical analysis}

All data were analyzed with SPSS software (SPSS, Inc., Chicago, IL., Version 20.0) using the General Linear Model procedure, repeated measurement ANOVA procedure was used $(2 \times 3)$ to analyze the statistical difference between time-points, and t-test was used for difference within time-point. Differences between means were tested using Tukey's Multiple Comparison Range Test. All data were presented as means \pm standard error and probability values less than 0.05 were considered statistically significant. Considering the statistical model as follow:

$Y_{i j k}=\mu_{v}+\alpha_{i}+\beta_{j}+(\alpha \beta)_{i j}+\varepsilon_{i j k}$

Where,

$Y_{i j k}=$ Dependent variable.

$\mu_{v}=$ General mean.

$\alpha_{i}=$ Effect of the diet factor $(i=2)$.

$\beta_{j}=$ Effect of the weeks $(j=3)$.

$(\alpha \beta)_{i j}=$ Interaction between 2 factors.

$\varepsilon_{i j k}=$ Normally distributed random experimental error on repeated measures with variance.

Diets and supply time were 2 factors. Control diet and ISF diet are 2 diets, and the ISF supplementation period of 4, 12 and 20 wks were considered as 3 time points.

\section{RESULTS}

\section{Performance}

After feeding ISF for 20 weeks, the effects of group factor, week's factor and the interaction between groupx weeks were analyzed. No significant change was observed in body weight between the control group and ISF supplemented group within the timepoint. The ISF group at 44 wks showed a heavier body weight $(p<0.001)$ than 36 and 52 wk old hens. As shown in Table 2, the egg production rate and average egg mass showed no statistical differences between 
Table 2 - The main effect (mean \pm SE) of soy isoflavones supplementation on egg production rate, average egg weight, average egg mass, feed conversion ratio and average daily feed intake of Chinese local broiler breeder hens at 36, 44, and 52 weeks of age.

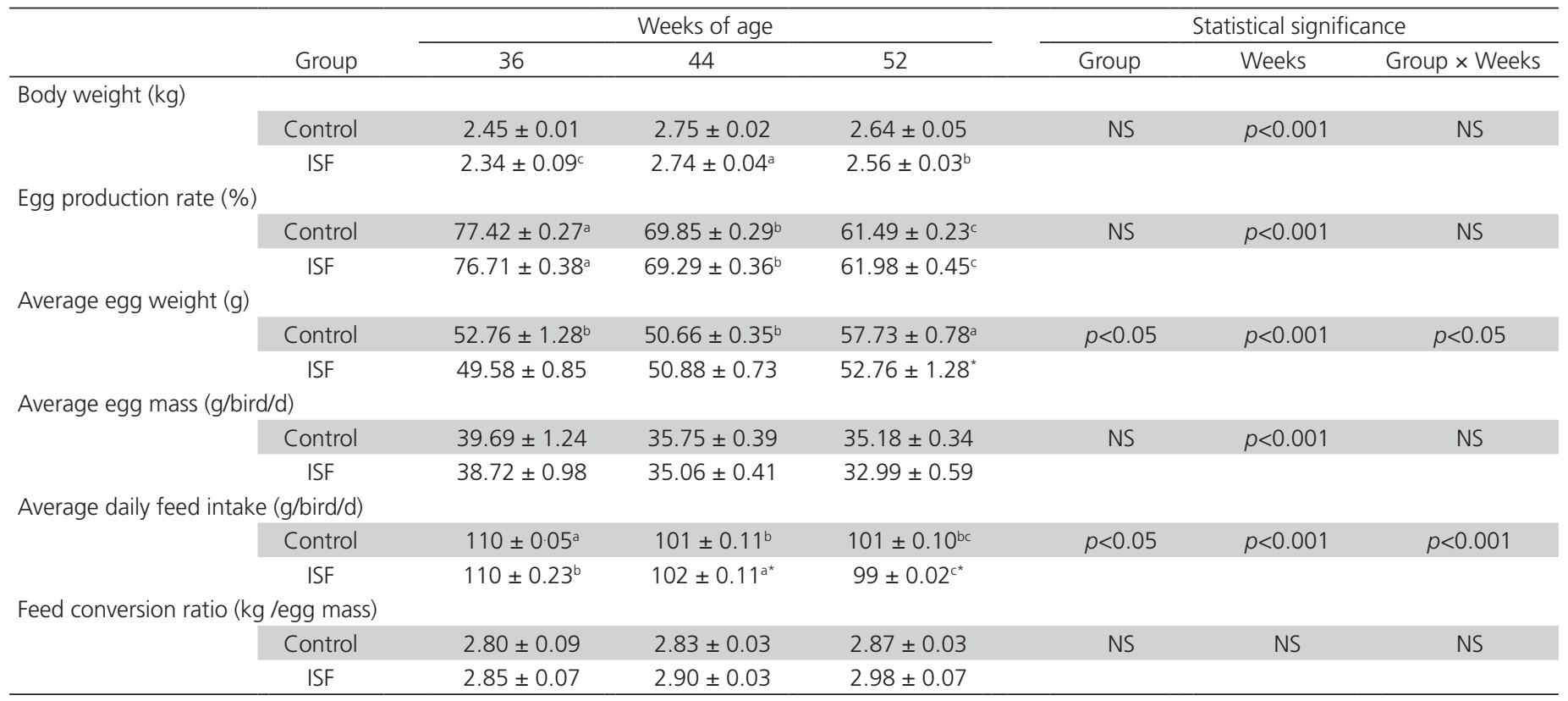

The number in each group was $n=8$. SE, standard error; ISF, soy isoflavones; NS, no significant difference.

"Means significantly different $(p<0.05)$ in the same column.

a-cDifferent superscripts in the same row mean differ significantly $(p<0.05)$.

the control and treatment group within the time interval. A significant reduction in both egg production rate $(p=0.018)$ and egg mass were observed with age increase within time interval investigated. At 52 wk of age, the ISF significantly decreased the egg weight in comparison to the control group ( $p=0.02)$. Among the control groups, at different weeks, the $52 \mathrm{wk}$ old hens had a heavier egg weight than at 36 and 44 wks $(p=0.01)$. A significant interaction between group $\times$ weeks was observed in average egg weight $(p=0.018)$ and average daily feed intake $(p=0.0001)$.

\section{Fertility and hatchability}

As shown in Table 3, no significant difference was observed between the control and treatment groups at 36,44 , and 52 wks of age in soft egg rate. The hatching rate improved significantly $(p=0.01)$ in ISF supplemented group at 36 wks of age compared

Table 3 - The main effect (mean \pm SE) of soybean isoflavones on hatching rate, abnormal egg rate, and soft egg rate of Chinese local broiler breeder hens at 36, 44, and 52 weeks of age.

\begin{tabular}{|c|c|c|c|c|c|c|c|}
\hline & \multirow[b]{2}{*}{ Group } & \multicolumn{3}{|c|}{ Weeks of age } & \multicolumn{3}{|c|}{ Statistical significance } \\
\hline & & 36 & 44 & 52 & Group & Weeks & Group $\times$ Weeks \\
\hline \multicolumn{8}{|l|}{ Hatching rate (\%) } \\
\hline & Control & $97.65 \pm 0.12^{b}$ & $97.93 \pm 0.0^{\mathrm{ab}}$ & $98.12 \pm 0.09^{a}$ & $p<0.05$ & $p<0.05$ & $p<0.001$ \\
\hline & ISF & $98.39 \pm 0.11^{a^{*}}$ & $97.78 \pm 0.12^{b}$ & $97.65 \pm 0.14^{b}$ & & & \\
\hline \multicolumn{8}{|c|}{ Abnormal egg rate (\%) } \\
\hline & Control & $1.25 \pm 0.13^{\mathrm{a}}$ & $0.68 \pm 0.05^{b c}$ & $0.79 \pm 0.00^{b}$ & $p<0.05$ & $p<0.05$ & $p<0.001$ \\
\hline & ISF & $0.66 \pm 0.11^{*}$ & $0.78 \pm 0.04$ & $0.83 \pm 0.06$ & & & \\
\hline \multicolumn{8}{|l|}{ Soft egg rate (\%) } \\
\hline & Control & $1.22 \pm 0.04$ & $1.36 \pm 0.05$ & $1.31 \pm 0.00$ & NS & $p<0.05$ & NS \\
\hline & ISF & $1.16 \pm 0.06$ & $1.52 \pm 0.11$ & $1.45 \pm 0.09$ & & & \\
\hline \multicolumn{8}{|l|}{ Fertility (\%) } \\
\hline & Control & $94.50 \pm 0.43$ & $93.50 \pm 0.43$ & $92.33 \pm 0.33$ & NS & $p<0.001$ & NS \\
\hline & ISF & $94.33 \pm 0.33$ & $93.83 \pm 0.48$ & $92.00 \pm 0.45$ & & & \\
\hline
\end{tabular}

The number in each group was $n=8$; SE, standard error; ISF, soy isoflavones; NS, no significant difference.

"Means significantly different $(p<0.05)$ in the same column.

${ }^{a-c}$ Different superscripts in the same row mean differ significantly $(p<0.05)$. 
with that recorded in the control group. However, a significant decrease was observed due to the increase in age ( $p=0.029)$. The ISF decreased abnormal egg rate in 36 wks of age compared to the untreated group $(p=0.02)$. No significant difference was observed between control and treatment groups at 36, 44, and 52 wks of age in fertility rate. The interaction between treatment and duration has a significant effect on hatchability rate and abnormal egg rate $(p=0.001)$.

\section{Egg quality}

The egg quality parameters were shown in Table 4. The ISF significantly increased the egg index percentage at $36(p=0.036)$ and 52 wks of age $(p=0.02)$ respectively, but not at $44 \mathrm{wks}$. The ISF treated group had lower HU compared to the control group at 36 wks of age $(p=0.032)$, while ISF supplementation significantly increased $\mathrm{HU}$ compared with the control group at 52 wks of age $(p=0.039)$. At the time-points of 36 wks of age, the control group showed heavier shell weight than at peak period of the treated group. Regarding the internal egg quality, the eggs from ISF at 44 wks of age showed higher yolk color $(p=0.035)$ than the control group, while the eggs from 52 wks of age had lower yolk color than the control group $(p=0.01)$. At late laying period, the treated group has a significant reduction $(p=0.017)$ in albumen weight compared with that recorded from the control group at the same age. A significant interaction between treatment $\times$ weeks was observed in index percentage, $\mathrm{HU}(p=0.032)$, yolk color $(p=0.018)$, albumen height $(p=0.02)$ and yolk weight $(p=0.002)$.

Table 4 - The main effect (mean \pm SE) of supplemented soy isoflavones on some egg quality parameters of Chinese local broiler breeder hens at 36,44 , and 52 weeks of age.

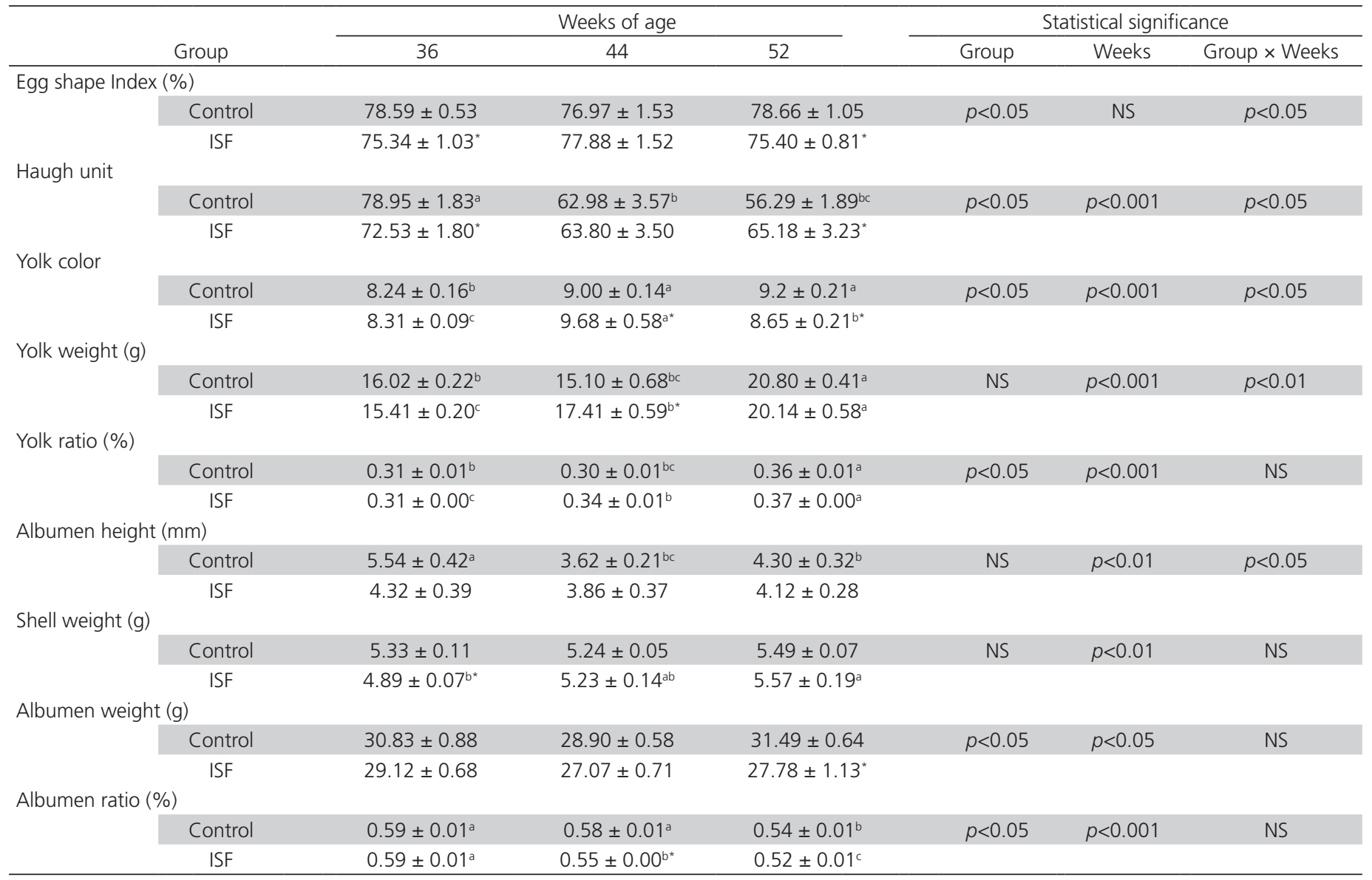

The number in each group was $n=8$. SE, standard error; ISF, soy isoflavones; NS, no significant difference.

"Means significantly different $(p<0.05)$ in the same column.

${ }^{a-c}$ Different superscripts in the same row mean differ significantly $(p<0.05)$.

\section{Organ relative weight}

The relative hens' organs weights are shown in Table 5. A significant increase was observed in relative liver weight between the control groups at 44 wks of age compared 52 wks of age $(p=0.045)$ respectively. In addition, ISF inclusion has a significant increase in 
Table 5 - The main effect $(m e a n \pm S E)$ of soy isoflavones supplementation on relative organs weight of Chinese local broiler breeder hens at 36,44 , and 52 weeks of age.

\begin{tabular}{|c|c|c|c|c|c|c|c|}
\hline & \multirow[b]{2}{*}{ Group } & \multicolumn{3}{|c|}{ Weeks of age } & \multicolumn{3}{|c|}{ Statistical significance } \\
\hline & & 36 & 44 & 52 & Group & Weeks & Group $\times$ Weeks \\
\hline \multicolumn{8}{|l|}{ Relative heart (\%) } \\
\hline & Control & $0.41 \pm 0.00^{a}$ & $0.47 \pm 0.03^{a}$ & $0.33 \pm 0.01^{b}$ & NS & NS & NS \\
\hline & ISF & $0.42 \pm 0.02$ & $0.39 \pm 0.03$ & $0.34 \pm 0.02$ & & & \\
\hline \multicolumn{8}{|l|}{ Relative liver (\%) } \\
\hline & Control & $2.12 \pm 0.13$ & $1.98 \pm 0.14$ & $1.78 \pm 0.05$ & NS & $p<0.05$ & NS \\
\hline & ISF & $2.01 \pm 0.07^{\mathrm{ab}}$ & $2.13 \pm 0.04^{a}$ & $1.85 \pm 0.03^{b}$ & & & \\
\hline \multicolumn{8}{|l|}{ Relative spleen (\%) } \\
\hline & Control & $0.09 \pm 0.00^{\mathrm{a}}$ & $0.08 \pm 0.00^{\mathrm{ab}}$ & $0.07 \pm .00^{\mathrm{b}}$ & $p<0.05$ & $p<0.01$ & NS \\
\hline & ISF & $0.09 \pm 0.00^{a}$ & $0.07 \pm 0.00^{b}$ & $0.09 \pm 0.00^{\mathrm{a}^{*}}$ & & & \\
\hline \multicolumn{8}{|l|}{ Relative ovary (\%) } \\
\hline & Control & $1.48 \pm 0.06$ & $1.92 \pm 0.17$ & $1.96 \pm 0.11$ & NS & NS & NS \\
\hline & ISF & $1.89 \pm 0.11$ & $1.80 \pm 0.11$ & $2.07 \pm 0.21$ & & & \\
\hline \multicolumn{8}{|l|}{ Relative oviduct (\%) } \\
\hline & Control & $2.08 \pm 0.08$ & $2.06 \pm 0.09$ & $2.22 \pm 0.13$ & NS & NS & NS \\
\hline & ISF & $2.23 \pm 0.10$ & $2.08 \pm 0.17$ & $2.13 \pm 0.14$ & & & \\
\hline \multicolumn{8}{|l|}{ Oviduct length $(\mathrm{cm})$} \\
\hline & Control & $65.33 \pm 2.17$ & $59.88 \pm 2.41$ & $61.70 \pm 1.28$ & NS & NS & NS \\
\hline & ISF & $62.67 \pm 2.53$ & $62.17 \pm 2.39$ & $59.08 \pm 0.84$ & & & \\
\hline
\end{tabular}

The number in each group was $n=8 ; S E$, standard error; ISF, soy isoflavones; NS, no significant difference.

"Means significantly different $(p<0.05)$ in the same column.

a-c Different superscripts in the same row mean differ significantly $(p<0.05)$.

spleen relative weight compared to the control group at 52 wks of age. No significant interaction between ISF supplementation and time-point was observed throughout the experimental duration.

\section{Serum concentrations of some metabolites and antioxidant parameters}

As shown in Table 6, ISF dietary inclusion significantly decreased the serum level of TCHO at 52 wks compared to the control group. Among treated weeks groups, TCHO was higher in 44 and 52 wks compared to 36 wks. The interaction between treatment and time-point was observed $(p=0.0027)$. The ISF supplementation reduced serum level of LDL-C significantly $(p=0.05)$ in the late laying period of treated hens. ISF supplementation significantly elevated the T-AOC in all time-point compared to control groups. No significant interaction effect was observed between group and weeks in LDL-C, HDL-C, $\mathrm{TG}$, and T-AOC.

\section{DISCUSSION}

Phytoestrogens; as soybean ISF (daidzein, genistein, glycitein), was used as a feed additive for the biological functions. They exert controversial results in animal studies. However, the effect depended on several factors, including the dose, the form of ISF, duration of ISF intake and the breed or species. Our study has demonstrated the effects of $3 \mathrm{mg} / \mathrm{kg}$ ISF dose on performance, egg quality, hatchability, and some oxidative status in Chinese Xueshan local broiler breeder hens at different stages of its production cycle, peak, post-peak and terminal stage. In our study, no significant changes were noted regarding breeder hen's body weight or organs relative weight throughout the experimental period. Except for spleen relative weight which was significantly heavier at late laying period of treated group; this result may indicate that ISF might help in hen's immunity, which was in accordance with the dietary inclusion of $1-5 \%$ ISF for Japanese quail in the study by Wilhelmset al. (2006), and Goliomytis et al. (2014) who reported that phytoestrogen quercetin did not influence growth performance of broiler. In disagreement with Jiang et al. (2007) who reported that dietary supplementation with $10,20 \mathrm{mg} / \mathrm{kg}$ ISF significantly increased final body weight, daily weight gain and average feed intake in the male broiler. The reason for this may be due to restricted feeding program used and dosages. The experimental breeder hens started laying eggs at 20 wks of age. The influence of broiler breeder feeds on subsequent egg production performance (Peebles et al., 2000), and embryo development and egg hatchability (Peebles et al., 2000), have been well documented. In the present trial, age-related decrease in egg production was observed 
Table 6 - The main effect (mean \pm SE) of soybean isoflavones supplementation on serum concentration of some metabolites and antioxidant parameters of Chinese local broiler breeder hens at 36, 44, and 52 weeks of age.

\begin{tabular}{|c|c|c|c|c|c|c|c|}
\hline & \multirow[b]{2}{*}{ Group } & \multicolumn{3}{|c|}{ Weeks of age } & \multicolumn{3}{|c|}{ Statistical significance } \\
\hline & & 36 & 44 & 52 & Group & Weeks & Group $\times$ Weeks \\
\hline \multicolumn{8}{|l|}{$\mathrm{TCHO}(\mathrm{mmol} / \mathrm{l})$} \\
\hline & Control & $7.40 \pm 0.50^{a}$ & $6.46 \pm 0.41^{b}$ & $8.26 \pm 0.43^{a}$ & $p<0.05$ & $p<0.05$ & $p<0.05$ \\
\hline & ISF & $5.59 \pm 0.67^{b}$ & $7.72 \pm 0.52^{\mathrm{a}}$ & $6.54 \pm 0.34^{\mathrm{a}^{*}}$ & & & \\
\hline \multicolumn{8}{|l|}{ LDL-C (mmol//) } \\
\hline & Control & $6.33 \pm 1.24^{a}$ & $4.26 \pm 0.90^{b}$ & $9.58 \pm 1.28^{a}$ & $p<0.01$ & $p<0.05$ & NS \\
\hline & ISF & $5.48 \pm 0.43$ & $2.99 \pm 0.99$ & $5.33 \pm 0.82^{*}$ & & & \\
\hline \multicolumn{8}{|l|}{ HDL-C (mmol/l) } \\
\hline & Control & $0.47 \pm 0.13^{b}$ & $0.41 \pm 0.06^{b}$ & $1.30 \pm 0.25^{\mathrm{a}}$ & NS & $p<0.05$ & NS \\
\hline & ISF & $0.87 \pm 0.31$ & $0.45 \pm 0.02$ & $0.66 \pm 0.21$ & & & \\
\hline \multicolumn{8}{|l|}{$\mathrm{TG}(\mathrm{mmol} / \mathrm{l})$} \\
\hline & Control & $19.27 \pm 1.66$ & $20.10 \pm 0.40$ & $22.89 \pm 2.16$ & NS & NS & NS \\
\hline & ISF & $19.19 \pm 1.69$ & $20.36 \pm 1.50$ & $23.98 \pm 1.59$ & & & \\
\hline \multicolumn{8}{|l|}{ T-AOC (U/ml) } \\
\hline & Control & $4.36 \pm 0.24^{a}$ & $1.85 \pm 0.37^{b}$ & $2.07 \pm 0.23^{b}$ & $p<0.01$ & $p<0.001$ & NS \\
\hline & ISF & $6.09 \pm 0.27^{a^{*}}$ & $3.64 \pm 0.46^{b^{*}}$ & $3.43 \pm 0.16^{b^{*}}$ & & & \\
\hline
\end{tabular}

The number in each group was $\mathrm{n}=8$; SE, standard error; TCHO, total cholesterol; LDL-C, low-density lipoprotein cholesterol; HDL-C, high -density lipoprotein cholesterol; TG, triglyceride; T-AOC, total anti-oxidative capability; ISF, soy isoflavones; NS, no significant difference.

"Means significantly different $(p<0.05)$ in the same column.

a-c Different superscripts in the same row mean differ significantly $(p<0.05)$.

in both groups. Egg production rate, egg mass, and feed conversion ratio were not influenced due to ISF supplementation throughout the experimental period. It was consistent with the previous results reported by Lu et al. (2017) using high ISF dosage in laying diet, and in contrast with several studies (Zhao et al., 2005; Ni et al., 2007; Gu et al., 2013; Khalaji et al., 2013; Shi et al., 2013), this might be due to the breed and ISF dosage. However, a significant reduction in the average daily feed intake was recorded in a late laying stage; consequently; a reduction in the average egg weight was observed at the same time-point. In opposite with results reported by Zhao et al. (2005), and Zhao et al. (2013) who used ISF daidzein to feed duck and geese respectively.

Many factors can affect the hatchability rate, such as different treatment during storage and incubation. However, it is suggested that ISF influence hatchability and fertility by improving eggshell quality, especially in older breeder hens. In this experiment, we found that ISF reduced the abnormal egg rate and hatchability rate at peak laying stage and significantly lowered the hatchability rate in the late production stage. In agreement with Zhao et al. (2013), who supplemented ISF daidzein to the geese diet, and in contrast with, (Zhao et al., 2004; Zhao et al., 2005). However, these conflicting results might be due to the phytoestrogen source used in the other study, dosage, duration, and the species.
Age factor has a direct impact on some internal and external egg quality parameters, and it is well documented that egg weight increased with hens' age, leading to heavier shell, yolk and albumen weights. However, Zita et al. (2009)_ENREF_23 reported that proportional shell and albumen weight decrease with age, while the yolk proportion weight increased with age. Also, De Matos (2008)_ENREF_36 reported that $\mathrm{Ca}$ provides and support the structural strength of bones and eggshell. Moreover, a study by Messina et al.(2004) showed that higher consumption of isoflavones increased bone density, and there is a tight link between Ca and ISF (Fonseca \& Ward, 2004). Subsequently, the availability of Ca for eggshell formation increased due to the ability of ISF to decrease intracellular Ca concentration in osteoclasts (Kajiya et al., 2000). In the present study, ISF supplementation enhanced egg quality parameters as follows: yolk weight, $\mathrm{HU}$, the percentage of egg index, shell weight, and yolk ratio, inconsistent with previous results (Sahin et al., 2007; Akdemir \& Sahin, 2009; Mattioli et al., 2017). In contrast, Zhao et al. (2005) claimed that feeding ISF daidzein increased albumen weight and decreased yolk weight in Shaoxing duck.

ISF has hypocholesterolemic effects in humans and in animals (Carroll \& Kurowska, 1995; Yang et al., 2012). In the present study, ISF treatment was lowering the serum level of TCHO and LDL-C during late laying period (52 wks of age), but without a change in HDL-C 
and TG, this was in agreement with a previous study (Taku et al., 2007). Also, no change was observed in TCHO and TG serum level when a high level of ISF daidzein was used (Lu et al., 2017), while Torres et al. (2006) noticed a redution in the serum level concentration of Cholesterol and TG in rats. Potter et al. (1998) suggested that ISF might have an estrogenic effect in the lowering of LDL and elevation of $\mathrm{HDL}$ concentration, which might be result from stimulation of bile acid excretion and increased liver LDL cholesterol receptor expression (Anderson et al., 1995).

The T-AOC is a useful tool to measure the redox status since it considers the cumulative action of all antioxidants (Ghiselli et al., 2000). ISF exhibit their antioxidant activity by donating a hydrogen atom from the hydroxyl group (Sierens et al., 2002). In this trail, ISF treatment showed an elevation in serum T-AOC throughout the experiment, which was in agreement with previous studies (Jiang et al., 2007; Liu et al., 2009; Kamboh \& Zhu, 2013).

\section{CONCLUSION}

In conclusion, dietary ISF supplementation for 20 weeks improved yolk weight and yolk color at post-peak and $\mathrm{HU}$ at the terminal stage with LDL-C reduction in the same period of Xueshan breeder hens, which indicated that the egg quality was improved by ISF diet. The body weight was not affected by ISF diet, however, ISF administration elevated T-AOC in the serum throughout the breeder laying cycle and increased relative spleen weight, which suggest that the antioxidant and immunity conditions might be improved by ISF diet. Thus, ISF could be used as a potential alternative of a synthetic feed additive to enhance healthy status for broiler breeder hens.

\section{REFERENCES}

Ajdžanović VZ, Medigović IM, Pantelić JB, Milošević VL. Soy isoflavones and cellular mechanics. Journal of Bioenergetics and Biomembranes 2014;46(2):99-107

Akdemir F, Sahin K. Genistein supplementation to the quail: effects on egg production and egg yolk genistein, daidzein, and lipid peroxidation levels. Poultry Science 2009;88(10):2125-2131

Anderson TJ, Uehata A, Gerhard MD, Meredith IT, Knab S, Delagrange D, Lieberman EH, Ganz et al. Close relation of endothelial function in the human coronary and peripheral circulations. Journal of the American College of Cardiology 1995;26(5):1235-1241.

Carroll KK, Kurowska EM. Soy consumption and cholesterol reduction: review of animal and human studies. The Journal of Nutrition 1995;125(suppl3):594S-597S.

Cassidy. Potential risks and benefits of phytoestrogen-rich diets. International Journal for Vitamin and Nutrition Research 2003;73(2):120-126.
De Matos R. Calcium metabolism in birds. Veterinary Clinics of North America: Exotic Animal Practice 2008;11(1):59-82.

Fonseca D, Ward WE. Daidzein together with high calcium preserve bone mass and biomechanical strength at multiple sites in ovariectomized mice. Bone 2004;35(2):489-497.

Ghiselli A, Serafini M, Natella F, Scaccini C. Total antioxidant capacity as a tool to assess redox status: critical view and experimental data. Free Radical Biology and Medicine 2000;29(11):1106-1114

Goliomytis M, Tsoureki D, Simitzis PE, Charismiadou MA, Hager-Theodorides $\mathrm{AL}$, Deligeorgis SG. The effects of quercetin dietary supplementation on broiler growth performance, meat quality, and oxidative stability. Poultry Science 2014;93(8):1957-1962.

Gu H, Shi S, Chang L, Tong H, Wang Z, Zou J. Safety evaluation of daidzein in laying hens: Part II. Effects on calcium-related metabolism. Food and Chemical Toxicology 2013;55:689-692.

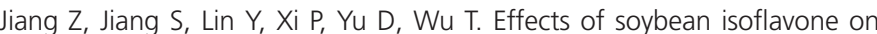
growth performance, meat quality, and antioxidation in male broilers. Poultry Science 2007;86(7):1356-1362.

Kajiya H, Okabe K, Okamoto F, Tsuzuki T, Soeda H. Protein tyrosine kinase inhibitors increase cytosolic calcium and inhibit actin organization as resorbing activity in rat osteoclasts. Journal of Cellular Physiology 2000;183(1):83-90.

Kamboh A, Zhu W-Y. Effect of increasing levels of bioflavonoids in broiler feed on plasma anti-oxidative potential, lipid metabolites, and fatty acid composition of meat. Poultry Science 2013;92(2):454-461.

Khalaji S, Zaghari M, Ganjkhanloo M, Ghaziani F. Arginine, soy isoflavone and hydroxypropyl methylcellulose have protective effects against obesity in broiler breeder hens fed on high-energy diets. British Poultry Science 2013;54(6):766-779.

Kontecka H, Nowaczewski S, Sierszuła MM. Analysis of changes in egg quality of broiler breeders during the first reproduction period. Annals of Animal Science 2012;12(4):609-620.

Kudou S, Fleury Y, Welti D, Magnolato D, Uchida T, Kitamura K, Okubo $\mathrm{K}$. Malonyl isoflavone glycosides in soybean seeds(Glycine max Merrill). Agricultural and Biological Chemistry1991;55(9):2227- 2233.

Liu HW, Gai F, Gasco L, Brugiapaglia A, Lussiana C, Guo KJ, et al. Effects of chestnut tannins on carcass characteristics, meat quality, lipid oxidation and fatty acid composition of rabbits. Meat Science 2009;83(4):678683.

Lu J, Qu L, Shen M, Li S, Dou T, Hu Y, et al. Safety evaluation of daidzein in laying hens: Effects on laying performance, hatchability, egg quality, clinical blood parameters, and organ development. Poultry Science 2017:96(7):2098-2103

Mattioli S, Ruggeri S, Sebastiani B, Brecchia G, Dal Bosco A, Mancinelli $A C$, et al. Performance and egg quality of laying hens fed flaxseed: highlights on n-3 fatty acids, cholesterol, lignans and isoflavones. Animal 2017;11(4):705-712.

Messina M, Ho S, Alekel DL. Skeletal benefits of soy isoflavones: a review of the clinical trial and epidemiologic data. Current Opinion in Clinical Nutrition \& Metabolic Care 2004;7(6):649-658.

Messina M. A brief historical overview of the past two decades of soy and isoflavone research. The Journal of Nutrition 2010;140(7):1350S-1354S.

Ni Y, Zhu Q, Zhou Z, Grossmann R, Chen J, Zhao R. Effect of dietary daidzein on egg production, shell quality, and gene expression of ER- $\alpha, G H-R$, and IGF-IR in shell glands of laying hens. Journal of Agricultural and Food Chemistry 2007;55(17):6997-7001. 
Ni YD, Wu J, Tong HY, Huang YB, Lu LZ, Grossmann R, et al. Effect of dietary daidzein supplementation on egg laying rate was associated with the change of hepatic VTG-II mRNA expression and higher antioxidant activities during the post-peak egg laying period of broiler breeders. Animal Feed Science and Technology 2012;177(1-2):116-123.

Peebles E, Zumwalt C, Doyle S, Gerard P, Latour M, Boyle C, et al. Effects of breeder age and dietary fat source and level on broiler hatching egg characteristics. Poultry Science 2000;79(5):698-704.

Peebles ED, Zumwalt C, Doyle S, Gerard P, Latour M, Boyle C, et al. Effects of dietary fat type and level on broiler breeder performance. Poultry Science 2000;79(5):629-639.

Pilsakova L, Riecanský I, Jagla F. The physiological actions of isoflavone phytoestrogens. Physiological Research 2010;59(5):651.

Potter SM, Baum JA, Teng H, Stillman RJ, Shay NF, Erdman J. Soy protein and isoflavones: their effects on blood lipids and bone density in postmenopausal women. The American Journal of Clinical Nutrition 1998;68(6):1375S-1379S.

Ratna WN. Inhibition of estrogenic stimulation of gene expression by genistein. Life Sciences 2002;71(8):865-877.

Sahin N, Onderci M, Balci T, Cikim G, Sahin K, Kucuk O. The effect of soy isoflavones on egg quality and bone mineralisation during the late laying period of quail. British Poultry Science 2007;48(3):363-369.

Shi S, Gu H, Chang L, Wang Z, Tong H, Zou J. Safety evaluation of daidzein in laying hens: Part I. Effects on laying performance, clinical blood parameters, and organs development. Food and Chemical Toxicology 2013;55:684-688.

Shin J-H, Park J-M, Bak D-J, Jean W-M, Song J-C, Kim S-K, et al. Effects of germinated and fermented unmarketable soybean on laying performance and egg quality in laying hens. Korean Journal for Food Science of Animal Resources 2008;28(5):667-674.

Sierens J, Hartley J, Campbell M, Leathem A, Woodside J. In vitro isoflavone supplementation reduces hydrogen peroxide-induced DNA damage in sperm. Teratogenesis, Carcinogenesis, and Mutagenesis 2002;22(3): 227- 234.

Taku K, Umegaki K, Sato Y, Taki Y, Endoh K, Watanabe S. Soy isoflavones lower serum total and LDL cholesterol in humans: a meta-analysis of 11 randomized controlled trials. The American Journal of Clinical Nutrition 2007;85(4):1148-1156
Torres N, Torre-Villalvazo I, Tovar AR. Regulation of lipid metabolism by soy protein and its implication in diseases mediated by lipid disorders. The Journal of Nutritional Biochemistry 2006;17(6):365-373.

Wilhelms K, Scanes C, Anderson L. Lack of estrogenic or antiestrogenic actions of soy isoflavones in an avian model: the Japanese quail. Poultry Science 2006;85(11):1885-1889.

Yang LL, Zhou QJ, Wang Y, Gao Y, Wang YQ. Comparison of the therapeutic effects of extracts from Spirulina platensis and amnion membrane on inflammation-associated corneal neovascularization. International Journal of Ophthalmology 2012;5(1):3 2-37.

Yousef MI, Esmail AM, Baghdadi HH. Effect of isoflavones on reproductive performance, testosterone levels, lipid peroxidation, and seminal plasma biochemistry of male rabbits. Journal of Environmental Science and Health, Part B 2004;39(5-6):819-833.

Zhang YZ, Shi DX, Zhou BC, Zeng CK, Pang SJ. Study on the Structure of C-Phycocyanin in Spirulina platensis with Scanning Tunneling Microscope. Sheng Wu Hua Xue Yu Sheng Wu Wu Li Xue Bao(Shanghai) 1997;29(5):521-525.

Zhao R, Wang Y, Zhou Y, Ni Y, Lu L, Grossmann R, et al. Dietary daidzein influences laying performance of ducks(Anas platyrhynchos) and early post-hatch growth of their hatchlings by modulating gene expression. Comparative Biochemistry and Physiology Part A: Molecular \& Integrative Physiology 2004;138(4):459-466.

Zhao R, Zhou Y, Ni Y, Lu L, Tao Z, Chen W, et al. Effect of daidzein on egglaying performance in Shaoxing duck breeders during different stages of the egg production cycle. British Poultry Science 2005;46(2):175-181.

Zhao X, Shao T, Wang Y, Lu X, Luo J, Zhou W. The phytoestrogen daidzein may affect reproductive performance of Zhedong White geese by regulating gene mRNA levels in the HPG axis. British Poultry Science 2013;54(2):252- 258

Zita L, Tůmová E, Štolc L. Effects of genotype, age and their interaction on egg quality in brown-egg laying hens. Acta Veterinaria Brno 2009;78(1):85-91. 
